\title{
Quantities and antibiotic resistance of microorganisms in some microbial products for animals in Vietnam
}

\author{
Nhi T. T. Nguyen, Ngoc H. Le, \& Hoa T. K. Ho*
}

Faculty of Animal Science and Veterinary Medicine, Nong Lam University, Ho Chi Minh City, Vietnam

\author{
ARTICLE INFO \\ Research Paper \\ Received: March 29, 2021 \\ Revised: May 31, 2021 \\ Accepted: June 10, 2021
}

\section{* Corresponding author}

Ho Thi Kim Hoa

Email: hoa.hothikim@hcmuaf.edu.vn

\begin{abstract}
The aims of the study were to look into the quantities of live beneficial microorganisms and antibiotic resistance of bacterial strains in several probiotic products used for food animals in the market. Ten probiotic products that claim to contain beneficial bacteria and fungi were examined. Eight products are said on the label to contain Lactobacillus spp., nine contain Bacillus spp., five contain yeasts and two have molds. The results showed that eight products did not have the microbial quantities or/and composition of microorganisms as saying on their labels. Of eight products which claim to contain Lactobacillus spp., the bacteria were isolated from only four, of which three had Lactobacillus counts at least ten-fold as low as the numbers on the labels. Spore-forming bacilli were isolated from all nine Bacillus-containing products. However, two products had the bacterial counts at least 10-fold as low as the numbers printed on the labels. Among five products stated to contain yeasts, the organisms were recovered from samples of only one. Seven Lactobacillus and fifteen Bacillus isolates from all samples that had bacterial growth were tested for their susceptibility against seven common antibiotics using Kirby-Bauer disk diffusion method. The results revealed that all the Lactobacillus isolates showed susceptibility to the tested antibiotics except kanamycin. All 15 Bacillus isolates were susceptible to ampicillin, kanamycin, and ciprofloxacin; five isolates were intermediately resistant to tetracycline; one isolates resisted erythromycin, and one isolates was resistant to vancomycin. The results of this study would provide information for farm practice in choosing antibiotics used together with antibiotics to maintain or/and restore the gut microflora after antibiotic treatment.
\end{abstract}

Cited as: Nguyen, N. T. T., Le, N. H., \& Ho, H. T. K. (2021). Quantities and antibiotic resistance of microorganisms in some microbial products for animals in Vietnam. The Journal of Agriculture and Development 20(3), 26-31.

\section{Introduction}

In the last two decades, the use of probiotics has become more and more popular in foodanimal production. They are used for promoting animal health status and disease prevention, and for improvement of productivity. Probiotics have become one of the most potent alterna- tives to replace antibiotics in animal production. FAO/WHO has provided a definition of probiotics, which has been used as selection criteria for probiotic strains to be used in foods and dietary supplements. Probiotic strains for human use must be (i) sufficiently characterized; (ii) safe for the intended use; (iii) supported by at least one positive human clinical trial conducted ac- 
cording to generally accepted scientific standards; and (iv) alive in the product at an efficacious dose throughout shelf life (Binda et al., 2020). Although the selection of beneficial bacteria for use in animals is not as strict as in humans, those criteria are still applied when a strain is studied for probiotic potential. Although most selected probiotic strains are safe, probiotics may possess undesirable properties such as virulence factors and transferable antimicrobial resistance (Alayande et al., 2020).

The objectives of the present study were to look into the quantities of live beneficial microorganisms and the resistance to some antibiotics of bacterial strains in several probiotic products used for food animals in the market.

\section{Materials and Methods}

Ten different products (named from A to $\mathrm{J}$ ) containing beneficial microbes - Lactobacillus spp., Bacillus spp. and fungi were purchased from veterinary stores. They were all made in Vietnam and recommended to be used as supplements for food animals. Eight products are said on the label to contain Lactobacillus spp., nine contain Bacillus spp., five contain yeasts and two have molds.

\subsection{Enumeration and isolation of the benefi- cial microbes from probiotic products}

Ten-fold serial dilution of each sample was made by suspending one gram of each product into nine $\mathrm{ml}$ of sterilized MRS broth (de man, Rogosa, Sharpe; Oxoid, CM0359) or saline. Microbial enumeration was performed using conventional plate count method. One hundred $\mu \mathrm{L}$ of each dilution $\left(10^{-1}\right.$ to $\left.10^{-6}\right)$ was spread on an appropriate agar plate to each group of examined microorganisms as described later. The process (weighing sample, making dilutions and inoculation) was repeated twice. If no growth were detected from the lowest dilution $\left(10^{-1}\right)$ from one repeat, the process was done for the third time.

\subsubsection{Lactic acid bacteria}

MRS agar (de man, Rogosa, Sharpe; Oxoid; CM0361) was used for enumeration of lactobacilli (De Man et al., 1960). One hundred $\mu \mathrm{L}$ of each sample dilution in MRS broth was spread onto an MRS agar and incubated at $37^{\circ} \mathrm{C} / 48 \mathrm{~h}$ under anaerobic condition (ThermoFisher, AN0025).
For each sample, colonies on a plate that had a growth of 20 - 200 colonies were accounted. Three colonies from the counted plate were restreaked onto new MRS agar plates and anaerobically incubated at $37^{\circ} \mathrm{C} / 48 \mathrm{~h}$. Each isolate was microscopically examined for Gram reaction and cell morphology. Test for catalase production was conducted by dripping two drops of hydrogen peroxide $3 \%$ onto bacteria on a glass slide. Isolates that were Gram-positive rods, non-spore forming and did not produce catalase was confirmed as Lactobacillus bacteria. Two isolates from each sample were kept in 15\%-glycerin MRS broth at $-20^{\circ} \mathrm{C}$ for further studies.

\subsubsection{Bacillus spp.}

TSA agar (Tryptone soya agar; Oxoid, CM0131) were used for enumeration of Bacillus spp. (Gorsuch et al., 2019). One hundred $\mu \mathrm{L}$ of each sample dilution in saline was spread onto a TSA plate and aerobically incubated at $37^{\circ} \mathrm{C} / 48 \mathrm{~h}$. Typical colonies of Bacillus bacteria were large, wrinkled, and saw-edged. For each sample, colonies on a plate that had a growth of 20 - 200 colonies were accounted. Three colonies from the counted plate were re-streaked onto new TSA agar plates and aerobically incubated at $37^{\circ} \mathrm{C} / 24 \mathrm{~h}$. Each isolate was microscopically examined for Gram reaction and cell morphology. Bacillus cells were large positive rods of which some were seen with endospores.

\subsubsection{Yeasts and molds}

Sabouraud dextrose agar (Oxoid, CM0041) containing chloramphenicol $5 \mathrm{mg} / 100 \mathrm{~mL}$ was used for growing yeasts and molds (Ladiges et al., 1974). One hundred $\mu \mathrm{L}$ of each sample dilution in saline was spread onto a Sabouraud plate and aerobically incubated at $30^{\circ} \mathrm{C}$, and checked for fungal growth after one day, two days and a week. For each sample, colonies on a plate of 20 - 200 colonies were accounted, from which three colonies were re-streaked onto new agar sabouraud dextrose agar plates. Yeast colonies were large, smooth, raised, with even edges. Mold colonies was large, fuzzy, and pigmented. Two or three colonies from each sample was Gramstained (yeasts) or Giemsa-stained (mold) and examined under a microscope at magnification 400x (Figure 1). 


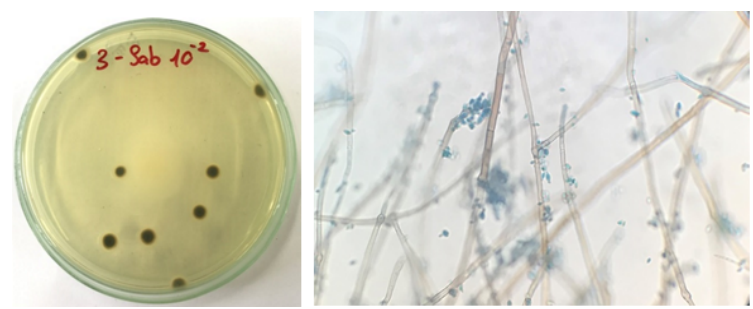

Figure 1. Mold colonies after one-day incubation (left) and Giemsa staining photo showing mold hyphae and spores (right; x400 magnification).

\subsection{Antibiotic susceptibility testing}

Two isolates of each microbe (Lactobacillus or Bacillus) from each sample (product) were tested for theirs susceptibility to seven antibiotics (ampicillin, amoxicillin/clavulanic, ciprofloxacin, erythromycin, kanamycin, tetracycline, vancomycin). The test was conducted using KirbyBauer disk diffusion method. The antibiotics belong to major groups which can be used for Grampositive bacteria. Briefly, suspension of each isolate was made with saline to obtain turbidity equivalent to McFarland scale 0.5. The suspensions of Lactobacillus isolates each was spread evenly onto an MRS agar plate with a sterile cotton swab. Bacillus isolates were spread on Mueller-Hinton agar (MHA, Oxoid, CM0337). Antibiotic discs (Nam Khoa Biotek, Vietnam) were then placed on the surface of inoculated plates. MRS plates were anaerobically incubated at $37^{\circ} \mathrm{C}$ for $48 \mathrm{~h}$ (Anisimova \& Yarullina, 2019). Plates of Bacillus isolates were aerobically incubated at $37^{\circ} \mathrm{C} / 24 \mathrm{~h}$ (Jang et al., 2018).

After the incubation, the diameter $(\mathrm{mm})$ of zone of inhibition (ZOI) was measured. Results were interpreted according to the recommendation by CLSI guidelines (Le \& Nguyen, 2016; Sharma et al., 2017). Isolates with ZOI $\geq 20 \mathrm{~mm}$ diameter were considered as susceptible (S); ZOI between 15 and $19 \mathrm{~mm}$ were as intermediate (I); and $\mathrm{ZOI} \leq 14 \mathrm{~mm}$ were resistant $(\mathrm{R})$.

\section{Results and Discussion}

\subsection{Enumeration and isolation of microorgan- isms}

\subsubsection{Lactobacillus spp.}

The results are presented in Table 1. Of those four products, the number of lactobacilli recov- ered from one product (E) was ten-fold as high as that said on the label; from two products (F \& G) it was more than ten-fold as low as the numbers on the labels; and from one product $(\mathrm{H})$ the bacterial count was at the lower range of that announced by the manufacturer.

\subsubsection{Bacillus spp.}

Of the nine samples that contain Bacillus spp., the numbers of bacteria recovered from four samples were similar as it said on the product labels (product B, D, H, I). The other five were not. From four samples (products E, F, G, J), bacterial counts were higher (approximately 1 to 3 $\log _{10} \mathrm{CFU} / \mathrm{g}$ ), whereas the count from sample $\mathrm{C}$ was $1 \log _{10} \mathrm{CFU}$ lower.

\subsubsection{Yeast and mold}

On the product labels, it was written that samples A and B contained both yeasts (Saccharomyces cerevisiae in A and Torulopsis bovina in B), and mold Aspergillus oryzae. However, the culture showed the growth of the molds but not the yeasts at all (after one week incubation). Furthermore, while the label of product A said that there were $2 \times 10^{6}$ Aspergillus oryzae $\mathrm{CFU} / \mathrm{g}$ of the product, the actual count was $4 \mathrm{x}$ $10^{3} \mathrm{CFU} / \mathrm{g}$. Similarly, it was $2 \times 10^{9}$ Aspergillus oryzae $\mathrm{CFU} / \mathrm{g}$ on the label of product $\mathrm{B}$, but the actual count was $8 \times 10^{6} \mathrm{CFU} / \mathrm{g}$. It meant the actual viable counts of the molds from two samples were about 1,000-fold as low as it was written on the labels. Three samples (D, H and J) said to have yeast species $S$. cerevisiae. However, no growth was recovered from two samples D and $\mathrm{H}$.

The enumeration and identification of probiotic microorganisms was carried out to evaluate the quality of the products which are available in the market. Four of the products claim to contained Lactobacillus spp., but none were recovered. There were five samples with revealed bacterial numbers were much lower than the numbers printed on product labels. In 2002, FAO/WHO gave a definition of probiotics as "live microorganisms which when administered in adequate amounts confer a health benefit on the host". In this document, it is recommended that minimum viable numbers of each probiotic strain at the end of the shelf-life should be described on the label. Of eight products saying to have Lactobacillus spp., only four did, of which the counts 
Table 1. Microbial counts from probiotic products (CFU/g)

\begin{tabular}{ccccc}
\hline \multirow{2}{*}{ Sample Lactobacillus spp. } & Counts & \multicolumn{2}{c}{ Bacillus spp. } \\
\cline { 2 - 5 } & Label $^{*}$ & nd & No & Counts \\
\hline $\mathrm{A}$ & $4.8 \times 10^{6}$ & $\mathrm{nd}$ & $2.0 \times 10^{7}$ & $4.4 \times 10^{7}$ \\
$\mathrm{~B}$ & $2.0 \times 10^{7}$ & $\mathrm{nd}$ & $5.0 \times 10^{6}$ & $5.0 \times 10^{5}$ \\
$\mathrm{C}$ & $5.0 \times 10^{6}$ & $\mathrm{nd}$ & $10^{6}$ & $8.2 \times 10^{5}$ \\
$\mathrm{D}$ & $10^{6}$ & $1.7 \times 10^{7}$ & $10^{6}$ & $8.0 \times 10^{8}$ \\
$\mathrm{E}$ & $10^{6}$ & $2.3 \times 10^{6}$ & $10^{5}$ & $5.1 \times 10^{7}$ \\
$\mathrm{~F}$ & $10^{5}$ & $8.6 \times 10^{3}$ & $10^{5}$ & $4.2 \times 10^{6}$ \\
$\mathrm{G}$ & 105 & $5.8 \times 10^{4}$ & $10^{4}-10^{7}$ & $8.3 \times 10^{6}$ \\
$\mathrm{H}$ & $10^{4}-10^{7}$ & - & $10^{8}$ & $9.3 \times 10^{7}$ \\
$\mathrm{I}$ & $\mathrm{No}$ & - & $5.6 \times 10^{5}$ & $5.0 \times 10^{6}$ \\
$\mathrm{~J}$ & $\mathrm{No}$ & &
\end{tabular}

${ }^{*}$ The numbers of microbes stated on product labels; nd: No growth from $100 \mu \mathrm{L}$ of 10 -fold sample dilution; CFU: Colony forming unit.

of three were at least ten-fold as low as those on the labels. On the other hands, Bacillus spp. were isolated from all nine samples, of which two had their counts at least ten-fold as low as they were supposed.

So, samples of four products A, B, C and D did not have the counts of all strains as it printed on the labels, either not detected or much less. Samples of products E, F, G had less numbers of Lactobacillus than the label stated, and sample of product $\mathrm{H}$ did not have yeast as it is mentioned in the label. In summary, eight out ten products did not have the target microbes or/and the numbers of at least one strain were lower than it described on the label.

The facts that Lactobacillus spp. were not recovered from samples of four products, while Bacillus spp. were isolated from all would indicate difficulties producing and maintaining the survival of the lactic-acid-bacteria species. This is probably due to the anaerobic property of Lactobacillus spp.. Although a majority are aerotolerant, optimal growth and survival require anaerobic conditions. In contract, Bacillus spp. are aerobes and spore-formers. Therefore, they can grow best in the air and survive harsh environments. Aerobic metabolism and spore forming are among main advantageous traits of Bacillus strains for their use as probiotics for farm animals and aquatic animals, when cost of production (that determines the price of the product) and the storage conditions, the way of application are important factors. There were four products that did not meet the statement (on the labels) about the presence and quantities of all beneficial strains. This is a worldwide problem about pro- biotic market. As reviewed by De Simone (2019), current regulation of probiotics is inadequate to protect consumers. So, the source of probiotics (manufactories and/or stores) should be considered if one want to buy a probiotic product.

\subsection{Antibiotic susceptibility of bacterial iso- lates}

\subsubsection{Antibiotic susceptibility of Lactobacillus isolates}

All seven isolates showed susceptibility to the tested antibiotics except kanamycin (Table 2). Among seven isolates of Lactobacillus, two were susceptible to kanamycin, one resistant and the other showed intermediate sensitivity to the antibiotic. This finding was consistent with some previous studies, which reported that lactobacilli were highly resistant to aminoglycosides (gentamycin, kanamycin, streptomycin) that act by inhibiting synthesis of protein (Gueimonde et al., 2013).

\subsection{Antibiotic susceptibility of Bacillus iso- lates}

The test was performed on 15 Bacillus isolates. The results are presented in Table 3 . All were susceptible to ampicillin, kanamycin, and ciprofloxacin. Five isolates showed intermediate susceptibility to tetracycline, one to amoxicillin/clavulanic and one to vancomycin. This results agreed with previous reports in the literature. For examples, $55.4 \%$ Bacillus cereus isolates from food samples by Tansuphasiri et al. (2006) and $33 \%$ of $B$. subtilis isolates from a study by 
Table 2. Antibiotic sensitivity of Lactobacillus isolates $(\mathrm{n}=7)$

\begin{tabular}{lcccccccc}
\hline \multirow{2}{*}{ Antibiotics } & $\begin{array}{c}\text { Disc concentration } \\
(\mu \mathrm{g})\end{array}$ & \multicolumn{7}{c}{ Isolates } \\
\cline { 3 - 9 } & 10 & $\mathrm{~L}$ & $6 \mathrm{~L}-1$ & $6 \mathrm{~L}-2$ & $9 \mathrm{~L}-1$ & $9 \mathrm{~L}-2$ & $10 \mathrm{~L}-1$ & $10 \mathrm{~L}-2$ \\
\hline Ampicillin & 30 & $\mathrm{~S}$ & $\mathrm{~S}$ & $\mathrm{~S}$ & $\mathrm{~S}$ & $\mathrm{~S}$ & $\mathrm{~S}$ & $\mathrm{~S}$ \\
Amoxicillin + Clavulanic & 30 & $\mathrm{~S}$ & $\mathrm{~S}$ & $\mathrm{~S}$ & $\mathrm{~S}$ & $\mathrm{~S}$ & $\mathrm{~S}$ & $\mathrm{~S}$ \\
Vancomycin & 15 & $\mathrm{~S}$ & $\mathrm{~S}$ & $\mathrm{~S}$ & $\mathrm{~S}$ & $\mathrm{~S}$ & $\mathrm{~S}$ & $\mathrm{~S}$ \\
Erythromycin & 30 & $\mathrm{~S}$ & $\mathrm{I}$ & $\mathrm{S}$ & $\mathrm{I}$ & $\mathrm{I}$ & $\mathrm{I}$ & $\mathrm{R}$ \\
Kanamycin & 30 & $\mathrm{~S}$ & $\mathrm{~S}$ & $\mathrm{~S}$ & $\mathrm{~S}$ & $\mathrm{~S}$ & $\mathrm{~S}$ & $\mathrm{~S}$ \\
Tetracycline & 5 & $\mathrm{~S}$ & $\mathrm{~S}$ & $\mathrm{~S}$ & $\mathrm{~S}$ & $\mathrm{~S}$ & $\mathrm{~S}$ & $\mathrm{~S}$ \\
Ciprofloxacin & & & & & & & &
\end{tabular}

S: Susceptible; I: Intermediate; R: Resistant.

Table 3. Antibiotic sensitivity of Bacillus isolates $(\mathrm{n}=15)$

\begin{tabular}{lccccccc}
\hline \multirow{2}{*}{ Antibiotics } & Disc concentration & \multicolumn{5}{c}{ Number of isolates } \\
\cline { 3 - 7 } & $(\mu \mathrm{g})$ & \multicolumn{3}{c}{$\mathrm{S}$} & \multicolumn{1}{c}{$\mathrm{I}$} & $\mathrm{R}$ \\
\hline Ampicillin & 10 & 15 & $100 \%$ & 0 & $0 \%$ & 0 & $0 \%$ \\
Amoxicillin + Clavulanic & 30 & 14 & $93.3 \%$ & 1 & $6.7 \%$ & 0 & $0 \%$ \\
Vancomycin & 30 & 13 & $86.6 \%$ & 1 & $6.7 \%$ & 1 & $6.7 \%$ \\
Erythromycin & 15 & 14 & $93.3 \%$ & 0 & $0 \%$ & 1 & $6.7 \%$ \\
Kanamycin & 30 & 15 & $100 \%$ & 0 & $0 \%$ & 0 & $0 \%$ \\
Tetracycline & 30 & 10 & $66.7 \%$ & 5 & $33.3 \%$ & 0 & $0 \%$ \\
Ciprofloxacin & 5 & 15 & $100 \%$ & 0 & $0 \%$ & 0 & $0 \%$ \\
\hline
\end{tabular}

S: Susceptible; I: Intermediate; R: Resistant.

Le \& Nguyen (2016) showed resistance to oxytetracycline.

Antibiotic resistance of probiotic bacteria can be either or both intrinsic or/and acquired. One of the main functions of probiotics in prevention or/and treatment gastrointestinal disorders in humans and animals is to maintain or/and restore the gut microflora after antibiotic treatment. Therefore, intrinsic antibiotic resistance could be useful. However, when the resistance determinants are located on mobile genetic elements or plasmids, it raises great concerns on public health. Those resistant probiotics can spread resistance genes to others in the gut microflora via horizontal gen transfer, hence creating a reservoir of resistance for potential food or gut pathogens (Sharma et al., 2014). Therefore, in recognition of the importance of assuring safety, determination of antibiotic resistance patterns of probiotic strains should be carried out (FAO/WHO, 2002). The results in this study did not find resistance to the seven common antibiotics among Lactobacillus isolates, and most Bacillus isolates were susceptible to the drugs except a number of isolates showing intermediate resistance to tetracycline. This would provide information for farm practice in choosing products containing beneficial bac- teria used together with antibiotics to maintain or/and restore the gut microflora after antibiotic treatment.

\section{Conclusions}

This was a small-scale study, which just looked at the quantities of microbial groups announced on the label, but not yet their benefits to animal health and productivity. Nevertheless, the results would provide some evidence for concerns about quality control of products containing beneficial microorganisms used for animals.

\section{References}

Alayande, K. A., Aiyegoro, O. A., \& Ateba C. N. (2020). Probiotics in animal husbandry: Application and associated risk factors. Sustainability 12(3), 1087.

Anisimova, E. A., \& Yarullina, D. R. (2019). Antibiotic resistance of Lactobacillus strains. Current Microbiology 76(12), 1407-1416.

Binda, S., Hill, C., Johansen, E., Obis, D., Pot, B., Sanders, M. E., Tremblay, A., \& Ouwehand, A. C. (2020). Criteria to qualify microorganisms as "probiotic" in foods and dietary supplements. Frontiers in Microbiology 11, 1662.

De Man, J. C., Rogosa M., \& Sharpe M. I. (1960). A 
medium for the cultivation of lactobacilli. Journal of Applied Bacteriology 23(1), 130-135.

De Simone, C. (2019). The unregulated probiotic market. Clinical Gastroenterology and Hepatology 17(5), 809817.

FAO/WHO (Food and Agriculture Organization/World Health Organization). (2002). Guidelines for the evaluation of probiotics in food. Paris, France: FAO.

Gorsuch, J. P., Jones, Z., Le Saint, D., \& Kitts, C. L. (2019). Enumeration of industrial Bacillus assemblages in commercial products with customized platecounting assays. Journal of Microbiological Methods 164, 105682.

Gueimonde, M., Sánchez, B., de los Reyes-Gavilán, C. G., \& Margolles, A. (2013). Antibiotic resistance in probiotic bacteria. Frontiers in Microbiology 4, 202.

Jang, K., Lee, J., Lee, H., Kim, S., Ha, J., Choi, Y., Oh, H., Yoon, Y., \& Lee, S. (2018). Pathogenic characteristics and antibiotic resistance of bacterial isolates from farmstead cheeses. Korean Journal for Food Science of Animal Resources 38(1), 203-208.
Ladiges, W. C., Foster, J. F., \& Jorgensen II, J. J. (1974). Comparison of media for enumerating fungi in precooked frozen convenience foods. Journal of Milk and Food Technology 37(6), 302-304.

Le, Y. T. H., \& Nguyen, H. D. (2016). Evaluation of the probiotic properties of Bacillus subtilis strains isolated from the Mekong Delta. Can Tho University Journal of Science 2, 26-32.

Sharma, C., Gulati, S., Thakur, N., Singh, B. P., Gupta, S., Kaur, S., Mishra, S. K., Puniya, A. K., Gill, J. P. S., \& Panwar, H. (2017). Antibiotic sensitivity pattern of indigenous lactobacilli isolated from curd and human milk samples. Biotech 7(1), 53.

Sharma, P., Tomar, S. K., Goswami, P., Sangwan, V., \& Singh, R. (2014). Antibiotic resistance among commercially available probiotics. Food Research International 57, 176-195.

Tansuphasiri, U., Khaminthakul, D., \& Pandii, W. (2006). Antibiotic resistance of enterococci isolated from frozen foods and environmental water. Southeast Asian Journal of Tropical Medicine and Public Health 37, 162-170. 